\begin{tabular}{lcccr} 
T H E & A R C H I V E & O F & M E C H A N I C A L & E N G I N E E R I N G \\
\hline VOL. LX & 2013 & Number 1
\end{tabular}

10.2478/meceng-2013-0006

Key words: continuum mechanics, rigid bodies, covariant, fluid-structure interaction

CHRISTIAN HESCH ${ }^{*}$, PETER BETSCH ${ }^{*}$

\title{
CONTINUUM MECHANICAL CONSIDERATIONS FOR RIGID BODIES AND FLUID-STRUCTURE INTERACTION PROBLEMS
}

\begin{abstract}
The present work deals with continuum mechanical considerations for deformable and rigid solids as well as for fluids. A common finite element framework is used to approximate all systems under considerations. In particular, we present a standard displacement based formulation for the deformable solids and make use of this framework for the transition of the solid to a rigid body in the limit of infinite stiffness. At last, we demonstrate how to immerse a discretized solid into a fluid for fluid-structure interaction problems.
\end{abstract}

\section{Introduction}

A mechanical system, independent of its physical interpretation, can often be considered as a continuum (see Malvern [12]). Within this continuum mechanical framework we can always formulate a local balance of momentum, either in terms of an Eulerian or a Lagrangian description. Since there is no general analytical solution for the initial boundary value problem under consideration, we apply a finite element solution method in space and time to approximate and solve the weak form of the problem, see Hughes [10].

If we consider bodies to be rigid, we can not achieve a numerical solution within a standard finite element framework, since the used degrees of freedom are not independent any more. Based on a specific Cosserat theory (see Rubin [13]) we can rewrite the finite element system in terms of a skew coordinate system. Within this reformulated system, we can incorporate the rigidity assumptions, such that we obtain a system of differential-algebraic equations (DAEs) governing the motion of the rigid body. As will be shown, the use

* Chair of Computational Mechanics, University of Siegen, Germany; E-mail: christian.hesch@uni-siegen.de 
CHRISTIAN HESCH, PETER BETSCH

of skew coordinate systems is necessary to account for interpolation errors of the director field.

The direct use of the introduced coordinate system yields a rotationless formulation which gives rise to this set of DAEs, see Betsch [5]. A further reduction of this DAEs to a minimum set of equations in terms of generalized coordinates is possible via a suitable null-space method (see Betsch [1]), and we recover the classical Newton-Euler equations.

In a last step we show how to immerse a solid in a fluid using the underlying finite element framework to create a suitable Euler-Lagrange mapping (Liu et al. [11] and Hesch et al. [8]). Thus, the solid is considered as a momentum source field arising within the fluid. This method is most preferable for fluid-structure interaction problems, since we do not have to remesh the fluid in each step. Similar techniques based on nodal force contributions instead of the field equations for the solid have been used to immerse particles (Hu et al. [9]).

The paper is organized as follows: The Lagrangian framework and the spatial discretization for solids is shown in Section 2. The transformation to a rigid body is demonstrated in Section 2.2. Fluid dynamics formulation is described in Section 2.3, whereas immersed techniques for fluid-structure interaction problems are presented in Section 3. Representative examples are shown in Section 4 and conclusions are drawn in Section 5.

\section{Continua}

In this section we provide a short outline of the fundamental equations under consideration. Developments concerning solids are based on a general non-linear approach within a Lagrangian framework written in its reference configuration $\mathcal{B}_{0} \subset \mathbb{R}^{3}$, whereas an Eulerian framework in the actual configuration $\mathcal{B} \subset \mathbb{R}^{3}$ is used for fluids. Subsection one summarizes the basic equations for a deformable, hyperelastic body, whereas subsection two modifies this formulation to embed the properties of a rigid body. Fluids are summarized in subsection three.

\subsection{Finite strain elastodynamics}

First, we consider a time-dependent deformation mapping $\varphi: \mathcal{B}_{0} \times$ $[0, T] \rightarrow \mathbb{R}^{3}$, where $[0, T]$ is the time interval elapsed during the motion. The corresponding mapping of the surface $\Gamma$ is denoted by $\gamma=\varphi(\Gamma, t)$. Note that we require that the boundaries satisfy

$$
\Gamma_{u} \cup \Gamma_{\sigma}=\Gamma \quad \text { and } \quad \Gamma_{u} \cap \Gamma_{\sigma}=\emptyset
$$


where $\Gamma_{u}$ and $\Gamma_{\sigma}$ denote the Dirichlet and Neumann boundaries, respectively. The current configuration is denoted by $\mathcal{B}_{t}=\varphi_{t}\left(\mathcal{B}_{0}\right)$. Material points are labeled by $\boldsymbol{X} \in \mathcal{B}_{0}$, the material velocity is given by $\boldsymbol{v}=\partial \varphi / \partial t$, and the deformation gradient is denoted by $\boldsymbol{F}=D \varphi$. Furthermore, we assume the existence of a strain energy function $\Psi(\boldsymbol{C}): \mathcal{B}_{0} \times[0, T] \rightarrow \mathbb{R}$, where $\boldsymbol{C}$ : $\mathcal{B}_{0} \times[0, T] \rightarrow \mathbb{R}^{3 \times 3}, \boldsymbol{C}=\boldsymbol{F}^{T} \boldsymbol{F}$ denotes the right Cauchy-Green deformation tensor. The linear momentum is given by $\pi=\varrho_{0} \boldsymbol{v}$, where $\varrho_{0}$ stands for the density in the reference configuration. The Lagrangian form of the balance of linear momentum is then given by

$$
\left[\begin{array}{l}
\dot{\varphi}=\rho_{0}^{-1} \pi \\
\dot{\pi}=\operatorname{Div}(\boldsymbol{P})+\overline{\boldsymbol{B}}
\end{array}\right]
$$

supplemented by the boundary conditions

$$
\begin{array}{rlll}
\varphi & =\bar{\varphi} & \text { on } & \Gamma_{u} \times[0, T] \\
\boldsymbol{P N}=\overline{\boldsymbol{T}} & \text { on } & \Gamma_{\sigma} \times[0, T]
\end{array}
$$

where $\boldsymbol{P}=2 \boldsymbol{F} \nabla_{\boldsymbol{C}} \Psi(\boldsymbol{C})$ denotes the first Piola-Kirchhoff stress tensor, $\overline{\boldsymbol{T}}$ the external tractions at the Neumann boundary and $\overline{\boldsymbol{B}}$ a body force. Furthermore, a superposed dot denotes the material time derivative. In the sequel we make use of the notation

$$
\int_{\mathcal{B}_{0}}(\bullet) \cdot(\bullet) d V=:\langle\bullet, \bullet\rangle \quad \text { and } \quad \int_{\Gamma}(\bullet) \cdot(\bullet) d A=:\langle\bullet, \bullet\rangle_{\Gamma}
$$

and express the contribution of a body to the virtual work as follows

$$
G(\boldsymbol{\varphi}, \delta \boldsymbol{\varphi})=\underbrace{\left\langle\rho_{R} \ddot{\boldsymbol{\varphi}}, \delta \boldsymbol{\varphi}\right\rangle}_{G^{\mathrm{dyn}}}+\underbrace{\left\langle\boldsymbol{P}, \nabla_{X}(\delta \boldsymbol{\varphi})\right\rangle}_{G^{\mathrm{int}}} \underbrace{-\left\langle\rho_{R} \overline{\boldsymbol{B}}, \delta \boldsymbol{\varphi}\right\rangle-\langle\overline{\boldsymbol{T}}, \delta \boldsymbol{\varphi}\rangle_{\Gamma_{\sigma}}}_{-G^{\mathrm{ext}}}
$$

To achieve a feasible numerical solution for the nonlinear problem under consideration, we apply a spatial discretization process to $\mathcal{B}_{0}$ by introducing a set of finite elements $e \in \mathbb{E}^{\text {h }}$ via

$$
\mathcal{B}_{0}^{\mathrm{h}}=\bigcup_{\forall e \in \mathbb{E}^{\mathrm{h}}} \mathcal{B}_{e}^{\mathrm{h}}
$$

Using a standard displacement-based finite element approach, we introduce finite dimensional approximations of $\varphi$ and $\delta \varphi$ given by

$$
\boldsymbol{\varphi}^{\mathrm{h}}=\sum_{A \in \omega} N^{A} \boldsymbol{q}_{A}, \quad \text { and } \quad \delta \boldsymbol{\varphi}^{\mathrm{h}}=\sum_{B \in \omega} N^{B} \delta \boldsymbol{q}_{B}
$$


where $\boldsymbol{q}_{A}=\varphi\left(X_{A}, t\right), A, B \in \omega=\left\{1, \ldots, n_{\text {node }}\right\}$ are the nodal values of the configuration mapping at time $t$, collected in a global configuration vector $\boldsymbol{q}=\left[\boldsymbol{q}_{1}, \ldots, \boldsymbol{q}_{n_{\text {node }}}\right]$. Furthermore, $N^{A}(\boldsymbol{X}): \mathcal{B}^{\text {h }} \rightarrow \mathbb{R}$ are the global shape functions associated with nodes $A$. The kinetic energy of the discrete system can now be written as

$$
T=\frac{1}{2}\left\langle\rho_{0} v^{\mathrm{h}}, v^{\mathrm{h}}\right\rangle
$$

Note that the coefficients of the corresponding discrete mass matrix read

$$
\boldsymbol{M}^{A B}=\left\langle\rho_{R} N^{A}, N^{B}\right\rangle \boldsymbol{I}
$$

where $\boldsymbol{I} \in \mathbb{R}^{3 \times 3}$ is the identity matrix. Next, we introduce the discrete deformation gradient and the discrete deformation tensor as follows

$$
\boldsymbol{F}^{\mathrm{h}}=\frac{\partial \varphi^{\mathrm{h}}}{\partial \boldsymbol{X}}=\sum_{A \in \omega} \boldsymbol{q}_{A} \otimes \nabla N^{A}(\boldsymbol{X})
$$

and

$$
\boldsymbol{C}^{\mathrm{h}}=\sum_{A, B \in \omega} \boldsymbol{q}_{A} \cdot \boldsymbol{q}_{B} \nabla N^{A}(\boldsymbol{X}) \otimes \nabla N^{B}(\boldsymbol{X})
$$

Based on the previously given definition of the local strain energy density function, the inner potential function reads

$$
V^{\text {int }}(\boldsymbol{q})=\left\langle\Psi\left(\boldsymbol{C}^{\mathrm{h}}\right), 1\right\rangle
$$

Thus, the discrete virtual work expression can be written as

$$
G^{\text {int }}\left(\varphi^{\mathrm{h}}, \delta \boldsymbol{\varphi}^{\mathrm{h}}\right)=\sum_{A, B \in \omega} \delta \boldsymbol{q}_{A} \cdot \boldsymbol{q}_{B}\left\langle\nabla N^{A}(\boldsymbol{X}), \boldsymbol{S}\left(\boldsymbol{C}^{\mathrm{h}}\right) \nabla N^{B}(\boldsymbol{X})\right\rangle
$$

where $\boldsymbol{S}\left(\boldsymbol{C}^{\mathrm{h}}\right)=2 \nabla \boldsymbol{C} \Psi\left(\boldsymbol{C}^{\mathrm{h}}\right)$ denotes the second Piola-Kirchhoff stress tensor. Furthermore, we assume the existence of an external potential energy function

$$
V^{\mathrm{ext}}(\boldsymbol{q})=\sum_{A \in \omega} \boldsymbol{q}_{A} \cdot\left(\left\langle N^{A}, \boldsymbol{B}\right\rangle+\left\langle N^{A}, \overline{\boldsymbol{T}}\right\rangle_{\Gamma_{\sigma}}\right)
$$

and we obtain for the discrete virtual work expression of the external contributions

$$
G^{\mathrm{ext}}\left(\boldsymbol{\varphi}^{\mathrm{h}}, \delta \boldsymbol{\varphi}^{\mathrm{h}}\right)=\delta \boldsymbol{q} \cdot \nabla V^{\mathrm{ext}}(\boldsymbol{q})
$$

Since (5) has to be valid for arbitrary test functions, we obtain a general nonlinear system of equations

$$
\left[\begin{array}{rl}
\dot{\boldsymbol{q}} & =\boldsymbol{v} \\
\boldsymbol{M} \dot{\boldsymbol{v}} & =-\left[\nabla V^{\mathrm{int}}(\boldsymbol{q})-\nabla V^{\mathrm{ext}}(\boldsymbol{q})\right]
\end{array}\right]
$$


which has to be solved using an adequate time stepping algorithm. Therefore, we split the considered time interval into a sequence of subintervals $n \rightarrow n+1$ and obtain

$$
\left[\begin{array}{rl}
\boldsymbol{q}_{n+1}-\boldsymbol{q}_{n} & =\Delta t \boldsymbol{v}_{n+1 / 2} \\
\boldsymbol{M}\left(\boldsymbol{v}_{n+1}-\boldsymbol{v}_{n}\right) & =-\Delta t\left[\bar{\nabla} V^{\mathrm{int}}\left(\boldsymbol{q}_{n+1}, \boldsymbol{q}_{n}\right)-\nabla V^{\mathrm{ext}}\left(\boldsymbol{q}_{n+1 / 2}\right)\right]
\end{array}\right]
$$

where $\bar{\nabla} V^{\text {int }}\left(\boldsymbol{q}_{n+1}, \boldsymbol{q}_{n}\right)$ represents the discrete gradient in the sense of Gonzalez [7]. Note that (17) conserves algorithmically energy and both momentum maps, see Betsch \& Steinmann [3].

\subsection{Rigid bodies}

Next, we want to constrain the previously introduced discrete and deformable system such that it remains rigid throughout the considered time interval. Based on the theory of Cosserat points (see Rubin [13]), we introduce a local coordinate system, attached to the body in the reference position at a specific point $\overline{\boldsymbol{X}}^{1}$ to measure the position of all mesh points in terms of convective coordinates $\theta^{i}$

$$
\boldsymbol{\varphi}^{\mathrm{h}}=\sum_{A \in \omega} N^{A} \boldsymbol{q}_{A}, \quad \boldsymbol{q}_{A}\left(\theta^{i}, t\right)=\bar{\varphi}(\overline{\boldsymbol{X}}, t)+\theta_{A}^{i} \boldsymbol{d}_{i}(t)
$$

Here, $\bar{\varphi}(\bar{X}, t) \in \mathbb{R}^{3}$ is the position vector of the reference point at time $t$ and $\boldsymbol{d}_{i}(t) \in \mathbb{R}^{3}$ constitute the director frame $\left\{\boldsymbol{d}_{1}, \boldsymbol{d}_{2}, \boldsymbol{d}_{3}\right\}$ at time $t$, which is not necessarily orthonormal. The basic kinematic assumptions for the rigidity of the body read

$$
\begin{array}{r}
\theta^{i} \neq \theta^{i}(t) \\
\boldsymbol{d}_{i} \cdot \boldsymbol{d}_{j}-\left(\boldsymbol{d}_{i} \cdot \boldsymbol{d}_{j}\right)_{\mathrm{ref}}=0
\end{array}
$$

i.e. the convective coordinates $\theta^{i}$ are not a function of time and the director triad does not deform. The motion of the rigid body is now characterized only by its kinetic energy and the constraints in (Eq. 19), since no internal stresses appear, i.e. $V^{\text {int }}(\boldsymbol{q})=0, \forall t \in[0, T]$. Analogues to (Eq. 8), we can now write for the kinetic energy

$$
T=\frac{1}{2} \sum_{e \in \mathbb{E}^{\mathrm{h}}}\left\langle\rho_{0} \boldsymbol{v}^{\mathrm{h}}, v^{\mathrm{h}}\right\rangle_{\boldsymbol{\theta}}^{e}
$$

\footnotetext{
${ }^{1}$ This point can, but do not have to be part of the body.
} 
using the velocity corresponding to (18)

$$
\boldsymbol{v}^{\mathrm{h}}=\sum_{A \in \omega} N^{A} \boldsymbol{v}_{A}, \quad \boldsymbol{v}_{A}\left(\theta^{i}, t\right)=\boldsymbol{v}_{\varphi}(\overline{\boldsymbol{X}}, t)+\theta_{A}^{i} \boldsymbol{v}_{i}(t)
$$

and the elementwise integration

$$
\langle\bullet, \bullet\rangle_{\boldsymbol{\theta}}^{e}=\int_{\mathcal{B}_{e}^{\mathrm{h}}}(\bullet) \cdot(\bullet) \operatorname{det}(\boldsymbol{R}) \mathrm{d} \boldsymbol{\theta}
$$

where $\boldsymbol{R}=\left[\begin{array}{lll}\boldsymbol{d}_{1} \boldsymbol{d}_{2} \boldsymbol{d}_{3}\end{array}\right], \boldsymbol{R} \in \mathbb{R}^{3 \times 3}$. For convenience, we introduce the extended position vector $\overline{\boldsymbol{q}}=\left[\overline{\boldsymbol{\varphi}}, \boldsymbol{d}_{1}, \boldsymbol{d}_{2}, \boldsymbol{d}_{3}\right]^{T}, \overline{\boldsymbol{q}} \in \mathbb{R}^{12}$ and obtain the corresponding constant mass matrix of the rigid body by inserting (22) into (21)

$$
\overline{\boldsymbol{M}}=\left[\begin{array}{cccc}
\boldsymbol{M}_{\varphi} & \boldsymbol{e}^{1} & \boldsymbol{e}^{2} & \boldsymbol{e}^{3} \\
\boldsymbol{e}^{1} & \boldsymbol{E}^{11} & \boldsymbol{E}^{12} & \boldsymbol{E}^{13} \\
\boldsymbol{e}^{2} & \boldsymbol{E}^{21} & \boldsymbol{E}^{22} & \boldsymbol{E}^{23} \\
\boldsymbol{e}^{3} & \boldsymbol{E}^{31} & \boldsymbol{E}^{32} & \boldsymbol{E}^{33}
\end{array}\right]
$$

where

$$
\begin{aligned}
\boldsymbol{M}_{\varphi} & =\sum_{e \in \mathbb{E}^{\mathrm{h}}}\left\langle\rho_{0}, 1\right\rangle_{\boldsymbol{\theta}}^{e} \boldsymbol{I}, \quad \boldsymbol{e}^{i}=\sum_{e \in \mathbb{E}^{\mathrm{h}}}\left\langle\rho_{0}, N^{A} \theta_{A}^{i}\right\rangle_{\boldsymbol{\theta}}^{e} \boldsymbol{I} \\
\boldsymbol{E}^{i j} & =\sum_{e \in \mathbb{E}^{\mathrm{h}}}\left\langle\rho_{0}, N^{A} N^{B} \theta_{A}^{i} \theta_{B}^{j}\right\rangle_{\boldsymbol{\theta}}^{e} \boldsymbol{I}
\end{aligned}
$$

Note that the inertia contributions $\boldsymbol{e}^{i}$ vanish, if $\bar{\varphi}(\bar{X}, t)$ is located at the center of mass of the body and that the matrix $\boldsymbol{E}$ will be diagonal, if the directors $\boldsymbol{d}_{i}$ are orthonormal and coincide with the principal axes of inertia.

Next, we rewrite the external contributions, assuming that the body force $\overline{\boldsymbol{B}}\left(\theta^{i}, t\right)$ and the Neumann boundary contributions $\overline{\boldsymbol{T}}\left(\theta^{i}, t\right)$ can be written in terms of the convective coordinates, such that

$$
G^{\mathrm{ext}}(\overline{\boldsymbol{q}}, \delta \overline{\boldsymbol{q}})=\sum_{e \in \mathbb{E}^{\mathrm{h}}}\left(\left\langle\delta \boldsymbol{\varphi}^{\mathrm{h}}, \overline{\boldsymbol{B}}\right\rangle_{\boldsymbol{\theta}}^{e}+\left\langle\delta \boldsymbol{\varphi}^{\mathrm{h}}, \overline{\boldsymbol{T}}\right\rangle_{\boldsymbol{\theta}, \Gamma_{\sigma}}^{e}\right)
$$

where $\delta \boldsymbol{\varphi}^{\mathrm{h}}=\sum_{A \in \omega} N^{A}\left(\delta \boldsymbol{\varphi}(\overline{\boldsymbol{X}}, t)+\theta_{A}^{i} \delta \boldsymbol{d}_{i}(t)\right)$. Rearranging all terms corresponding to the position vector $\overline{\boldsymbol{q}}$ yields

$$
G^{\mathrm{ext}}(\overline{\boldsymbol{q}}, \delta \overline{\boldsymbol{q}})=\delta \overline{\boldsymbol{q}} \cdot \nabla_{\overline{\boldsymbol{q}}} V^{\mathrm{ext}}(\overline{\boldsymbol{q}})=\delta \overline{\boldsymbol{q}} \cdot \overline{\boldsymbol{F}}, \quad \overline{\boldsymbol{F}}=\left[\boldsymbol{f}_{\varphi}, \boldsymbol{f}^{1}, \boldsymbol{f}^{2}, \boldsymbol{f}^{3}\right]^{T}
$$


where

$$
\begin{aligned}
\boldsymbol{f}_{\varphi} & =\sum_{e \in \mathbb{E}^{\mathrm{h}}}\left(\langle\overline{\boldsymbol{B}}, 1\rangle_{\boldsymbol{\theta}}^{e}+\langle\overline{\boldsymbol{T}}, 1\rangle_{\boldsymbol{\theta}, \Gamma_{\sigma}}^{e}\right) \\
\boldsymbol{f}^{i} & =\sum_{\boldsymbol{e} \in \mathbb{E}^{\mathrm{h}}}\left(\left\langle\overline{\boldsymbol{B}}, N^{A} \theta_{A}^{i}\right\rangle_{\boldsymbol{\theta}}^{e}+\left\langle\overline{\boldsymbol{T}}, N^{A} \theta_{A}^{i}\right\rangle_{\boldsymbol{\theta}, \Gamma_{\sigma}}^{e}\right)
\end{aligned}
$$

Note that we can associate Neumann contributions to the director momentum in terms of an external torque $\boldsymbol{m}$ using the kinematic relationship $\boldsymbol{v}_{i}=\boldsymbol{\omega} \times \boldsymbol{d}_{i}$, where $\omega \in \mathbb{R}^{3}$ denotes the angular velocity. Therefore, we calculate the power contributions arising from the external torque

$$
\boldsymbol{m} \cdot \omega=\frac{1}{2} \boldsymbol{m} \cdot\left(d^{i} \times v_{i}\right)=\frac{1}{2} v_{i} \cdot\left(\boldsymbol{m} \times d^{i}\right)=v_{i} \cdot f^{i}
$$

and obtain for the external director momentum

$$
\boldsymbol{f}^{i}=\frac{1}{2} \boldsymbol{m} \times \boldsymbol{d}^{i}
$$

Note that the consistent application of external torque (30) has originally been proposed in Betsch et al. [2]. At last, we derive the constraint forces based on (20), written as follows

$$
\Phi(\overline{\boldsymbol{q}})=\frac{1}{2}\left(\boldsymbol{d}_{i} \cdot \boldsymbol{d}_{j}-\left(\boldsymbol{d}_{i} \cdot \boldsymbol{d}_{j}\right)_{\mathrm{ref}}\right)
$$

and obtain the contributions of the constraint forces to the virtual work

$$
G^{\mathrm{con}}(\overline{\boldsymbol{q}}, \delta \overline{\boldsymbol{q}})=\delta \overline{\boldsymbol{q}} \cdot \overline{\boldsymbol{F}}^{\mathrm{con}}, \quad \overline{\boldsymbol{F}}^{\mathrm{con}}=\left[\boldsymbol{f}_{c}, \boldsymbol{f}_{c}^{1}, \boldsymbol{f}_{c}^{2}, \boldsymbol{f}_{c}^{3}\right]^{T}
$$

Since the constraints (31) do not depend on $\varphi(\bar{X}, t), \boldsymbol{f}_{c}=\mathbf{0}$. The remaining terms are given by

$$
\boldsymbol{f}_{c}^{i}=\nabla_{\boldsymbol{d}_{i}} \Phi(\overline{\boldsymbol{q}}) \cdot \lambda=\boldsymbol{\Lambda}^{i j} \boldsymbol{d}_{j}, \quad\left[\lambda^{i j}\right]=\left[\begin{array}{lll}
\lambda^{1} & \lambda^{4} & \lambda^{5} \\
\lambda^{4} & \lambda^{2} & \lambda^{6} \\
\lambda^{5} & \lambda^{6} & \lambda^{3}
\end{array}\right]
$$

where $\lambda^{i}$ denotes the Lagrange multipliers.

Analogues to (16), we obtain a nonlinear system of equations

$$
\left[\begin{array}{rl}
\dot{\overline{\boldsymbol{q}}} & =\overline{\boldsymbol{v}} \\
\overline{\boldsymbol{M}} \dot{\overline{\boldsymbol{v}}} & =-\left[\nabla_{\overline{\boldsymbol{q}}} \Phi(\overline{\boldsymbol{q}}) \cdot \lambda-\nabla_{\overline{\boldsymbol{q}}} V^{\mathrm{ext}}(\overline{\boldsymbol{q}})\right] \\
\Phi(\overline{\boldsymbol{q}}) & =\mathbf{0}
\end{array}\right]
$$


which again has to be solved by a suitable time stepping scheme. As before, we make use of a mid-point type rule (see Betsch \& Steinmann [4])

$$
\left[\begin{array}{rl}
\overline{\boldsymbol{q}}_{n+1}-\overline{\boldsymbol{q}}_{n} & =\Delta t \overline{\boldsymbol{v}}_{n+1 / 2} \\
\overline{\boldsymbol{M}}\left(\overline{\boldsymbol{v}}_{n+1}-\overline{\boldsymbol{v}}_{n}\right) & =-\Delta t\left[\nabla_{\overline{\boldsymbol{q}}} \Phi\left(\overline{\boldsymbol{q}}_{n+1 / 2}\right) \cdot \lambda-\nabla_{\overline{\boldsymbol{q}}} V^{\mathrm{ext}}\left(\overline{\boldsymbol{q}}_{n+1 / 2}\right)\right] \\
\Phi\left(\overline{\boldsymbol{q}}_{n+1}\right) & =\mathbf{0}
\end{array}\right]
$$

for the constrained system under consideration. The used type of a midpoint rule conserves algorithmically several mechanical properties such as total energy and the components of the angular momentum. Note that the constraints in (31) are only fulfilled at time $n+1$, not at $n+1 / 2$.

\subsection{Fluids}

To describe later on the interaction of fluids and structures, we start here by considering a finite element formulation of fluids. Therefore, we write the fluid system in terms of an Eulerian description using the inverse mapping $\boldsymbol{X}=\boldsymbol{\varphi}^{-1}(\boldsymbol{x}(t), t)$. The time differential of a physical quantity $f(\boldsymbol{x}(t), t)$ follows immediately

$$
\left.\frac{\partial f}{\partial t}\right|_{X}=\left.\frac{\partial f}{\partial t}\right|_{x}+v \cdot \nabla_{x} f
$$

Without loss of generality we restrict ourself to the incompressible case and obtain the continuity condition

$$
\nabla_{x} \cdot v=\frac{1}{J} \dot{J} \equiv 0
$$

where $J=\operatorname{det}(\boldsymbol{F})$. For a Newtonian fluid the Cauchy stress tensor $\boldsymbol{\sigma}$ : $\mathcal{B} \times[0, T] \rightarrow \mathbb{R}^{d \times d}$ is defined by

$$
\sigma=-p \boldsymbol{I}+\mu\left(\nabla_{x} v+\nabla_{x} v^{T}\right)
$$

Here, the pressure $p: \mathcal{B} \times[0, T] \rightarrow \mathbb{R}$ is a sufficiently smooth function and can be regarded as Lagrange multiplier to enforce (37). Furthermore, $\mu$ denotes the dynamic viscosity. The Eulerian form of the balance of linear momentum reads

$$
\rho \dot{\boldsymbol{v}}=\nabla_{\boldsymbol{x}} \cdot \boldsymbol{\sigma}+\rho \boldsymbol{b}
$$

where $\rho$ and $\boldsymbol{b}$ denote the density and a prescribed body force in the actual configuration, respectively. In weak form, the balance equation can be written as

$$
\langle\rho(\dot{\boldsymbol{v}}-\boldsymbol{b}), \delta \boldsymbol{v}\rangle_{\mathcal{B}}+\left\langle\boldsymbol{\sigma}, \nabla_{\boldsymbol{x}}(\delta \boldsymbol{v})\right\rangle_{\mathcal{B}}-\langle\boldsymbol{t}, \delta \boldsymbol{v}\rangle_{\gamma_{\sigma}}=0
$$


supplemented by the constraints

$$
\left\langle\nabla_{\boldsymbol{x}} \cdot \boldsymbol{v}, \delta p\right\rangle_{\mathcal{B}}=0
$$

Using a standard Galerkin based discretization of the velocity and the Lagrange multiplier field analogues to (Eq. 7), we obtain the semi-discrete balance of momentum

$$
\left\langle\rho^{f}\left(\dot{\boldsymbol{v}}^{\mathrm{h}}-\boldsymbol{b}^{\mathrm{h}}\right), \delta \boldsymbol{v}^{\mathrm{h}}\right\rangle_{\mathcal{B}}+\left\langle\boldsymbol{\sigma}^{f}\left(\boldsymbol{v}^{\mathrm{h}}, p^{\mathrm{h}}\right), \nabla_{\boldsymbol{x}}\left(\delta \boldsymbol{v}^{\mathrm{h}}\right)\right\rangle_{\mathcal{B}}-\left\langle\boldsymbol{t}^{\mathrm{h}}, \delta \boldsymbol{v}^{\mathrm{h}}\right\rangle_{\gamma^{\sigma}}=0
$$

and the semi-discrete incompressibility constraint

$$
\left\langle\nabla_{\boldsymbol{x}} \cdot \boldsymbol{v}^{\mathrm{h}}, \delta p^{\mathrm{h}}\right\rangle_{\mathcal{B}}=0
$$

If necessary (i.e. for low order elements or high Reynolds numbers), a stabilization technique for the underlying Galerkin approach can be applied (cf. Tezduyar [14]) using extended test function spaces

$$
\begin{aligned}
& \mathbb{V}^{\tilde{v}}=:\left\{\delta \tilde{\boldsymbol{v}} \in \mathbb{H}^{1}(\Omega) \mid \delta \tilde{\boldsymbol{v}}=\delta \boldsymbol{v}+\gamma_{\mathrm{SUPG}} \boldsymbol{v} \cdot \nabla_{\boldsymbol{x}}(\delta \boldsymbol{v})+\gamma_{\mathrm{PSPG}} \nabla_{\boldsymbol{x}}(\delta p)\right\} \\
& \mathbb{V}^{\tilde{p}}=:\left\{\delta \tilde{p} \in L_{2}(\Omega) \mid \delta \tilde{p}=\delta p+\gamma_{\mathrm{LSIC}} \nabla_{\boldsymbol{x}} \cdot(\delta \boldsymbol{v})\right\}
\end{aligned}
$$

where $\gamma_{\text {SUPG }}, \gamma_{\mathrm{PSPG}}$ and $\gamma_{\mathrm{LSIC}}$ are precalculated stabilization parameters. The modified semi-discrete balance of linear momentum reads

$$
\begin{aligned}
& \left\langle\rho^{f}\left(\dot{\boldsymbol{v}}^{\mathrm{h}}-\boldsymbol{b}^{\mathrm{h}}\right), \delta \boldsymbol{v}^{\mathrm{h}}\right\rangle_{\mathcal{B}}+\left\langle\boldsymbol{\sigma}^{f}\left(\boldsymbol{v}^{\mathrm{h}}, p^{\mathrm{h}}\right), \nabla_{\boldsymbol{x}}\left(\delta \boldsymbol{v}^{\mathrm{h}}\right)\right\rangle_{\mathcal{B}}-\left\langle\boldsymbol{t}^{\mathrm{h}}, \delta \boldsymbol{v}^{\mathrm{h}}\right\rangle_{\gamma^{\sigma}}+ \\
& \quad \sum_{e} \gamma_{\mathrm{SUPG}}\left\langle\mathcal{R}_{\boldsymbol{v}},\left(\boldsymbol{v}^{\mathrm{h}} \cdot \nabla_{\boldsymbol{x}}\right) \delta \boldsymbol{v}^{\mathrm{h}}\right\rangle_{\mathcal{B}}+\sum_{e} \gamma_{\mathrm{LSIC}}\left\langle\mathcal{R}_{p}, \nabla_{\boldsymbol{x}} \cdot \delta \boldsymbol{v}^{\mathrm{h}}\right\rangle_{\mathcal{B}}=0
\end{aligned}
$$

and the kinematic constraint

$$
\left\langle\nabla_{\boldsymbol{x}} \cdot \boldsymbol{v}^{\mathrm{h}}, \delta p^{\mathrm{h}}\right\rangle+\sum_{e} \gamma_{\mathrm{PSPG}}\left\langle\mathcal{R}_{v}, \nabla_{\boldsymbol{x}} \delta \boldsymbol{p}^{\mathrm{h}}\right\rangle_{\mathcal{B}}=0
$$

Here, $\mathcal{R}_{v}$ and $\mathcal{R}_{p}$ denote the residuals of the original momentum and kinematic constraint equations, respectively. The discretization in time of (42) and (43) using the previously introduced mid-point type rule, becomes

$$
\begin{aligned}
&\left\langle\rho \left(\frac{\boldsymbol{v}_{n+1}^{\mathrm{h}}-\boldsymbol{v}_{n}^{\mathrm{h}}}{\Delta t}-\left(\boldsymbol{v}_{n+1 / 2}{ }^{\mathrm{h}} \cdot \nabla_{\boldsymbol{x}}\right) \boldsymbol{v}_{n+1 / 2}^{\mathrm{h}}-\right.\right.\left.\left.\boldsymbol{g}_{n+1 / 2}^{\mathrm{h}}\right), \delta \boldsymbol{v}^{\mathrm{h}}\right\rangle+ \\
&\left\langle\boldsymbol{\sigma}\left(\boldsymbol{v}_{n+1 / 2}^{\mathrm{h}}, p_{n+1}^{\mathrm{h}}\right), \nabla_{\boldsymbol{x}}\left(\delta \boldsymbol{v}^{\mathrm{h}}\right)\right\rangle-\left\langle\boldsymbol{h}_{n+1 / 2}^{\mathrm{h}}, \delta \boldsymbol{v}^{\mathrm{h}}\right\rangle_{\Gamma^{h}}=0 \\
&\left\langle\nabla_{\boldsymbol{x}} \cdot \boldsymbol{v}_{n+1}^{\mathrm{h}}, \delta p^{\mathrm{h}}\right\rangle=0
\end{aligned}
$$

The fully discrete stabilized version follows in a straight forward manner and is given in detail in Hesch et al. [8]. 


\section{Fluid-Structure interaction}

For the calculation of fluid-structure interactions we immerse a solid system within the fluid (see Liu et al. [15]). To embed the resulting forces of the solid system, occupying the domain $\mathcal{B}_{t}^{s}$ at time $t$ as volumetric force $\mathcal{F}: \mathcal{B}_{t}^{s} \times[0, T] \rightarrow \mathbb{R}^{3}$ within the balance of linear momentum of the fluid, we reformulate (Eq. 39) as follows ${ }^{2}$

$$
\rho^{f} \dot{\boldsymbol{v}}=\nabla_{\boldsymbol{x}} \cdot \boldsymbol{\sigma}^{f}+\rho^{f} \boldsymbol{b}+\mathcal{F}
$$

The force field of the immersed solid reads

$$
\mathcal{F}=\left\{\begin{array}{lll}
\mathbf{0} & \text { in } & \mathcal{B} \backslash \mathcal{B}^{s} \\
\left(\rho^{f}-\rho^{s}\right)(\dot{\boldsymbol{v}}-\boldsymbol{b})+\nabla \cdot\left(\boldsymbol{\sigma}^{s}-\boldsymbol{\sigma}^{f}\right) & \text { in } & \mathcal{B}^{s}
\end{array}\right.
$$

We obtain the actual stress field of the solid via push forward of the purely material derivative of the strain energy function

$$
\boldsymbol{\sigma}^{s}=\frac{2}{J} \boldsymbol{F} \cdot \frac{\partial \Psi(\boldsymbol{C})}{\partial \boldsymbol{C}} \boldsymbol{F}^{T}
$$

Due to its physical properties it is convenient to use a Lagrangian mapping for the immersed solid, whereas the fluid uses an Eulerian mapping. This necessitates the definition of an Euler-Lagrange mapping $\mathcal{I}_{\mathcal{B}_{t}^{s}}$ for any given function $\psi$ of the solid system, occupying area $\mathcal{B}_{t}^{s} \subset \mathcal{B}$ such that $\psi(\boldsymbol{x}, t)$ : $\mathcal{B}_{t}^{s} \times[0, T]$ maps to $\mathcal{I}_{\mathcal{B}_{t}^{s}}(\psi(\boldsymbol{X}, t)): \mathcal{B}_{0}^{s} \times[0, T]$. This motivates the mapping

$$
\boldsymbol{v}(\boldsymbol{x}(t), t)=\mathcal{I}_{\mathcal{B}_{t}^{s}}(\boldsymbol{v}(X, t))
$$

To complete the set of equations we define appropriate Dirichlet boundary conditions for the immersed solid

$$
\boldsymbol{x}(\boldsymbol{X}, t)=\tilde{\boldsymbol{x}}, \quad \text { on } \quad \partial \mathcal{B}_{D}^{s}
$$

Since the immersed solid is surrounded by the fluid, additional Neumann boundary conditions are not treated explicitly. Thus, the corresponding weak form reads

$$
\left\langle\rho^{f}(\dot{\boldsymbol{v}}-\boldsymbol{b})-\mathcal{F}, \delta \boldsymbol{v}\right\rangle_{\mathcal{B}}+\left\langle\boldsymbol{\sigma}^{f}, \nabla_{\boldsymbol{x}}(\delta \boldsymbol{v})\right\rangle_{\mathcal{B}}-\langle\boldsymbol{t}, \delta \boldsymbol{v}\rangle_{\gamma^{h}}=0
$$

and for the constraints

$$
\left\langle\nabla_{\boldsymbol{x}} \cdot \boldsymbol{v}, \delta p\right\rangle_{\mathcal{B}}=0
$$

${ }^{2}$ Contributions to the solid system are marked with $(\bullet)^{s}$, whereas contributions to the fluid are marked with $(\bullet)^{f}$. 
The spatial discretization of the fluid follows analogues to (45), thus we focus here on the discretization of the volumetric force field of the immersed solid and obtain

$$
\begin{aligned}
\mathcal{F}^{\mathrm{h}}(\boldsymbol{v}, p)= & \left\langle\left(\rho_{0}^{f}-\rho_{0}^{s}\right)\left(\mathcal{I}_{\mathcal{B}_{t}^{s}}\left(\boldsymbol{v}^{\mathrm{h}}\right)-\boldsymbol{b}^{\mathrm{h}}\right), \mathcal{I}_{\mathcal{B}_{t}^{s}}\left(\delta \tilde{\boldsymbol{v}}^{\mathrm{h}}\right)\right\rangle_{\mathcal{B}_{0}}^{s} \\
& -\left\langle\boldsymbol{S}^{s},\left(\boldsymbol{F}^{s}\left(\mathcal{I}_{\mathcal{B}_{t}^{s}}\left(\boldsymbol{v}^{\mathrm{h}}\right)\right)\right)^{T} \nabla_{\boldsymbol{X}} \mathcal{I}_{\mathcal{B}_{t}^{s}}\left(\delta \tilde{\boldsymbol{v}}^{\mathrm{h}}\right)\right\rangle_{\mathcal{B}_{0}}^{s} \\
& +\left\langle\overline{\boldsymbol{\sigma}}^{f}\left(\mathcal{I}_{\mathcal{B}_{t}^{s}}\left(\boldsymbol{v}^{\mathrm{h}}\right), \mathcal{I}_{\mathcal{B}_{t}^{s}}\left(p^{\mathrm{h}}\right)\right), \nabla_{\boldsymbol{x}} \mathcal{I}_{\mathcal{B}_{t}^{s}}\left(\delta \tilde{\boldsymbol{v}}^{\mathrm{h}}\right) J^{s}\left(\mathcal{I}_{\mathcal{B}_{t}^{s}}\left(\boldsymbol{v}^{\mathrm{h}}\right)\right)\right\rangle_{\mathcal{B}_{0}}^{s}
\end{aligned}
$$

where we use an IFEM approach for the Euler-Lagrange mapping

$$
\mathcal{I}_{\mathcal{B}_{t}^{s}}\left(N^{A}(\boldsymbol{X}, t)\right)=\sum_{C=1}^{n_{\text {node }}} N^{A}\left(\boldsymbol{x}\left(\boldsymbol{X}_{C}, t\right)\right) \bar{N}_{C}(\boldsymbol{X})
$$

Although tedious, the discretization in time is straight forward, see Hesch et al. [8].

\section{Numerical example}

This example deals with the application of immersed techniques to cardiovascular problems. Therefore, we consider blood as incompressible Newtonian viscous fluid with viscosity $\mu=1$ and density $\rho=1 \cdot 10^{5}$. Two flaps are inserted into the channel, see Fig. 1, the top and the bottom sides are fixed and a Poiseuille inflow is applied to the left using the amplitude function $A(t)=5 \cdot(\sin (2 \pi t)+1.1)$ and no boundary conditions are imposed to the right hand side. The flaps are modeled as Neo-Hookian solids using the Lamé parameters $\lambda^{s}=8 \cdot 10^{6}$ and $\mu^{s}=2 \cdot 10^{6}$ corresponding to a Young's modulus of $E=5.6 \cdot 10^{6}$ and a Poisson ratio of $v=0.4$. They can be regarded as idealization of a human heart valve exposed to insufficiency modeled by the gap between both flaps, cf. Gil et al. [6].

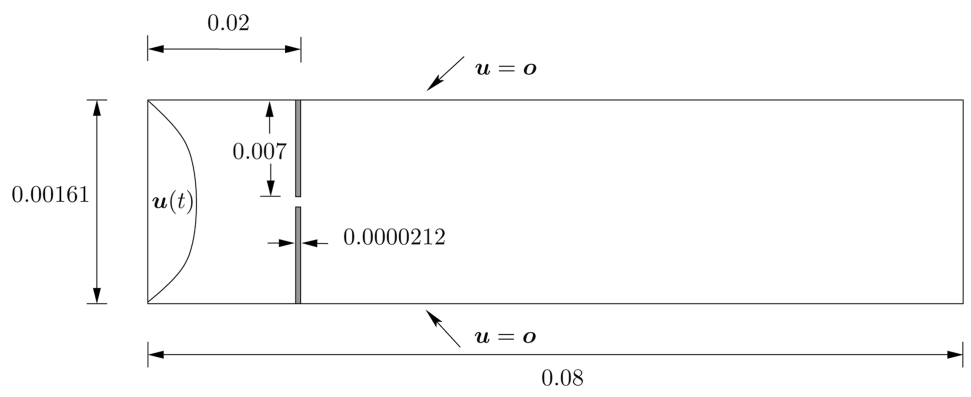

Fig. 1. Geometry and boundary conditions for two flapping membranes

The series of figures in Fig. 2 shows the time evolution for the pulsatile flow using 256x64 Q1Q1 fluid and 40x4 bilinear solid elements. 


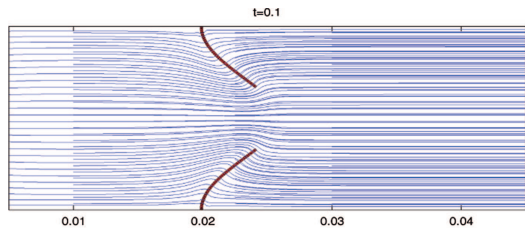

$t=0.3$

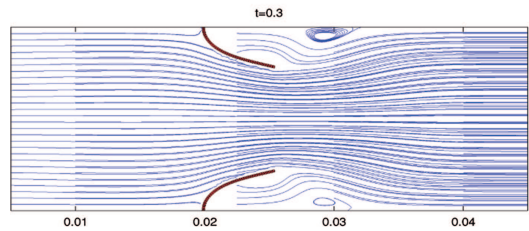

$\mathrm{t}=0.5$

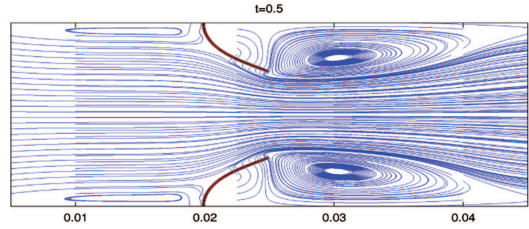

$\mathrm{t}=0.7$

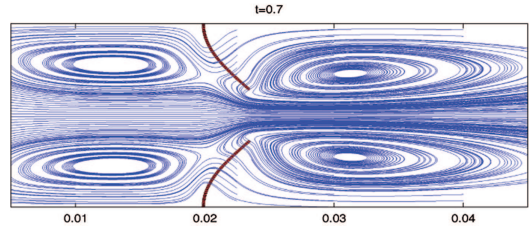

$\mathrm{t}=0.9$

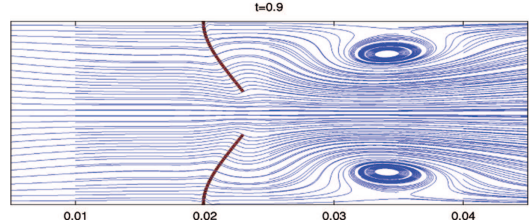

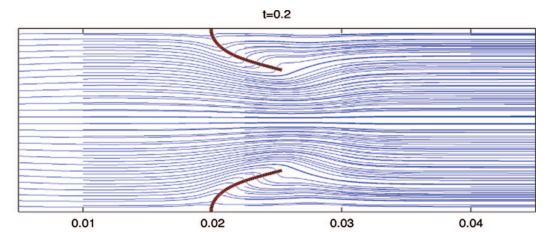

$\mathrm{t}=0.4$
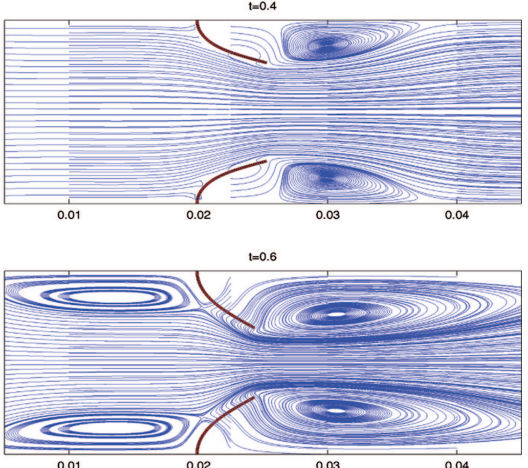

$t=0.8$

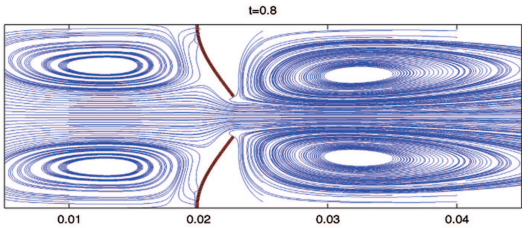

$t=1$

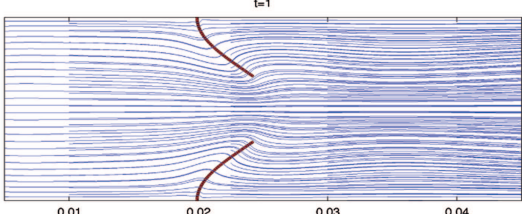

Fig. 2. Time evolution of the membranes and streamlines of the fluid

The movement of the tip of the upper leaflet in the $\mathrm{X}$ and $\mathrm{Y}$ directions are shown in Figures 3 and 4, respectively, for different discretizations. The results based on the Q1Q1 fluid finite element discretization converge to the results of the Q2Q1 fluid finite element discretization for a sufficiently fine mesh. The converged results are in perfect agreement with those obtained with the Immersed Structural Potential Method (ISPM) shown in Gil et al. [6]. A detailed discussion between these different immersed techniques can be found in Hesch et al. [8].

\section{Conclusions}

Based on a general continuum mechanical description, we have first introduced a non-linear framework for deformable bodies. In a second step, 


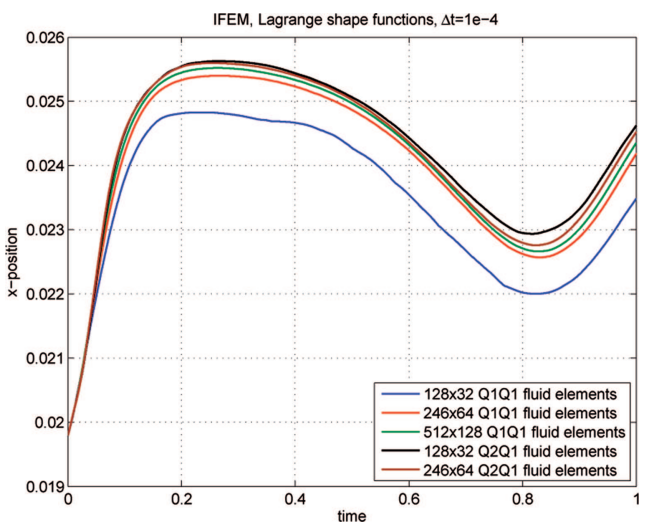

Fig. 3. X-Position of the tip of the upper leaflet

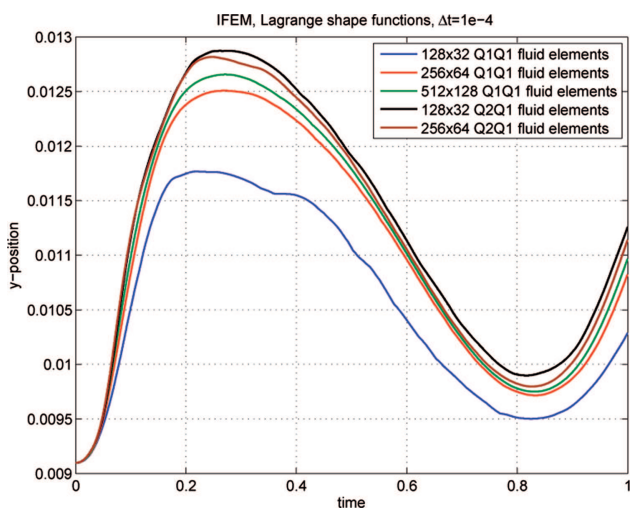

Fig. 4. Y-Position of the tip of the upper leaflet

a most general approach has been introduced to reformulate the discretized body in the case of infinite stiffness as a constrained system using a redundant set of coordinates. No restrictions are set to the coordinate system of the rigid body, such that we can use skew coordinate systems. This is of major importance, since we can now account for interpolation errors of the directors, if, for instance, we apply a mid-point type rule for the discretization in time.

In a last step, we have shown how to immerse a discrete system into a fluid, such that we can treat the immersed solid as momentum source field in the fluid. In a future work, we will transfer the rigid body assumptions to the immersed system as well, such that we can account for rigid bodies in a fluid in a concise and easy way. The foundations for this work are laid in this article. 


\section{REFERENCES}

[1] P. Betsch: The discrete null space method for the energy consistent integration of constrained mechanical systems Part I: Holonomic constraints. Comput. Methods Appl. Mech. Engrg., 194:5159-5190, 2005.

[2] P. Betsch, R. Siebert, N. Saenger: Natural coordinates in the optimal control of multibody systemssie. J. Comput. Nonlinear Dynam., 7(1):011009/1-8, 2011.

[3] P. Betschand, P. Steinmann: Conserving properties of a time FE method - Part II: Timestepping schemes for non-linear elastodynamics. Int. J. Numer. Methods Eng., 50:1931-1955, 2001.

[4] P. Betschand, P. Steinmann: Conservation Properties of a Time FE Method. Part III: Mechanical systems with holonomic constraints. Int. J. Numer. Methods Eng., 53:2271-2304, 2002.

[5] P. Betschand, S. Uhlar: Energy-momentum conserving integration of multibody dynamics. Multibody System Dynamics, 17(4):243-289, 2007.

[6] A.J. Gil, A. Arranz Carreño, J. Bonet, O. Hassan: The immersed structural potential method for haemodynamic applications. Journal of Computational Physics, 229:8613-8641, 2010.

[7] O. Gonzalez: Time integration and discrete Hamiltonian systems. J. Nonlinear Sci., 6:449-467, 1996.

[8] C. Hesch, A.J. Gil, A. Arranz Carreño, J. Bonet: On immersed techniques for fluid-structur interaction. Comput. Methods Appl. Mech. Engrg., 247-248:51-64, 2012.

[9] H.H. Hu, N.A. Patankar, M.Y. Zhu: Direct numerical simulations of fluid-soli systems using the arbitrary Lagrangian-Eulerian technique. Journal of Computational Physics, 169:427-462, 2001.

[10] T.J.R. Hughes: The Finite Element Method. Dover Publications, 2000.

[11] W.K. Liu, D.W. Kim, S. Tang: Mathematical foundations of the immersed finite lement method. Computational Mechanics, 39:211-222, 2007.

[12] L.E. Malvern: Introduction to the mechanics of a continous medium. Prentice Hall, 1969.

[13] M.B. Rubin: Cosserat Theories: Shells, Rodsand Points. Kluwer Academic Publishers, 2010.

[14] T.E. Tezduyar: Stabilized finit element for mulations for incompressible flow computations. Advances in applied mechanics, 28:1-44, 1992.

[15] L. Zhang, A. Gerstenberger, X. Wang, W.K. Liu: Immersed finit element method. Comput. Methods Appl. Mech. Engrg., 193:2051-2067, 2004.

\section{Koncepcja continuum w mechanice ciał sztywnych i problemach interakcji między płynem i strukturą}

\section{Streszczenie}

Przedstawiona praca dotyczy mechaniki continuum w zastosowaniu do ciał sztywnych i odkształcalnych oraz do płynów. W ramach wspólnego systemu elementów skończonych dokonano aproksymacji całego rozważanego systemu. W szczególności, przedstawiono standardowe, oparte na przemieszczeniach, sformułowanie FEM dla ciał deformowalnych i wykorzystano je przy przejściu granicznym do ciała o nieskończonej sztywności. W końcu, zademonstrowano problemy interakcji między płynem a strukturą na przykładzie zdyskretyzowanego ciała zanurzonego w cieczy. 